\title{
Modeling and Control of an Electric Arc Furnace
}

\author{
Benoit Boulet, Gino Lalli and Mark Ajersch \\ Centre for Intelligent Machines \\ McGill University \\ 3480 University Street, Montréal, Québec, Canada H3A 2A7
}

\begin{abstract}
Electric arc furnaces (EAFs) are widely used in steelmaking and in smelting of nonferrous metals. The EAF is the central process of the so-called mini-mills, which produce steel mainly from scrap. Typical EAFs operate at power levels from 10MW to $100 \mathrm{MW}$. The power level is directly related to production throughput, so it is important to control the EAF at the highest possible average power with a low variance to avoid breaker trips under current surge conditions. For efficient power control, good dynamic models of EAFs are required. This paper solves the electrical circuit of an EAF with a floating neutral, proposes a dynamic model for the EAF, and investigates simple proportional electrode current and power control.
\end{abstract}

\section{Introduction}

Electric arc furnaces (EAFs) are widely used in steelmaking and in smelting of nonferrous metals. The EAF is the central process of the so-called mini-mills, which produce steel mainly from scrap. Typical EAFs operate at power levels from 10MW to $100 \mathrm{MW}$. The power level is directly related to production throughput, so it is important to control the EAF at the highest possible average power with a low variance to avoid breaker trips under current surge conditions. For efficient power control, good dynamic models of EAFs are required [1].

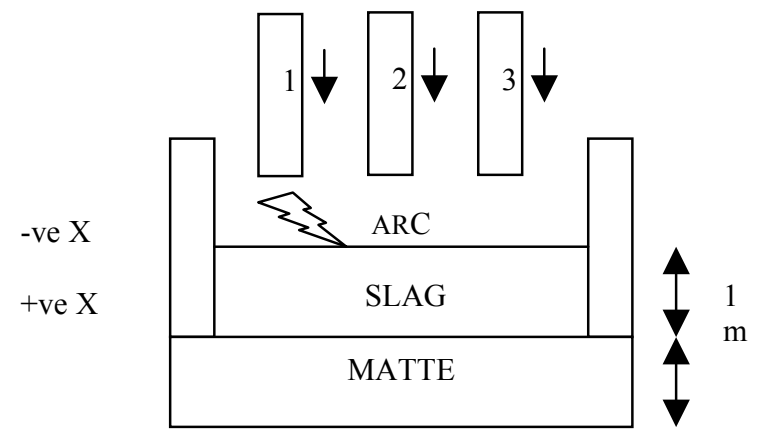

Figure 1: Physical Model of EAF

\section{Physical Model of Arc Furnace}

Figure 1 shows the physical model of the electric arc furnace. In this particular EAF model, there are three electrodes that are moved vertically up and down with hydraulic actuators. Each of these electrodes has a diameter of roughly $1.5 \mathrm{~m}$, weighs approximately 40 tons and is 1 to 2 stories tall. In theory, the ore is melted with a huge power surge from the electrodes. The actual product is denser than the scrap and thus falls to the bottom of the furnace creating the matte. Above the matte lies the slag where the electrode tips are dipped. The tremendous heat created by these electrodes causes the ore to liquefy and separate. Thereupon more raw materials are placed in the furnace and the process repeats itself.

\subsection{Arcing}

Arcing is a phenomenon that occurs when the electrodes are moved above the slag. As the electrode approaches the slag, current begins to jump from the electrode to the slag, creating electric arcs. Depending on the magnitude of the input voltages of the electrodes, the arcing distance can vary. Usually, arcing occurs in a region within centimeters of the slag (approximately 10$15 \mathrm{~cm})$. Therefore, the EAF model must take into account the instances when $\mathrm{x}_{1}, \mathrm{x}_{2}, \mathrm{x}_{3}$ are negative (i.e. the electrodes are suspended above the slag). Figure 1 above shows the sign convention used in the project.

\section{Solving the Electrical Circuit of the EAF}

To solve any electrical model, assumptions are made to facilitate the derivation. Similarly, the EAF electrical circuit requires several assumptions before reaching the final equations. The first step in the analysis of the electrical circuit is to use Kirchoff's Current Law (KCL) to equate currents and voltages. Figure 2 shows 4 nodes, one for each of the electrodes and the fourth representing the virtual ground at the matte $\left(\mathrm{V}_{\mathrm{m}}\right)$. Using these nodes, it is possible to determine the current in each electrode with respect to each voltage and the conductance coefficients, using its position as the input. Proper assumptions can facilitate derivations thus calculating the following equation involving matrices:

$$
\left[I_{i}\right]=\left[G_{i j}\right]\left[x_{i}\right]+\left[B_{i}\right]
$$

\section{Equation 1: Current Matrix Model}

Here, $I_{i}$ is a $3 \times 1$ matrix with electrode currents, $G_{i j}$ is a $3 \times 3$ conductance matrix and $\mathrm{B}_{\mathrm{i}}$ is a $3 \times 1$ constant matrix.

\subsection{Assumptions}

For the EAF circuit, several assumptions were made. It must be noted that this is a three-phase circuit with a double configuration. The outer resistances (inter-electrode resistances) form a deltacircuit with the three nodes. The inner resistances (slag-to-matte resistances) form a wye-connection with $\mathrm{V}_{\mathrm{m}}$ as a virtual ground (floating neutral). Figure 2 shows the electrical model for the EAF with the chosen direction of currents.

To simplify calculations, the inter-electrode resistances are equivalent and represented by $\mathrm{R}$. As for the slag-to-matte resistances, tests showed that these resistances displayed inverse linear relations with respect to their position. Consequently, by taking the slag-to-matte conductances, the inverse function becomes a linear relationship, which makes for simpler calculations. The slag-to-matte conductances $G_{i}$, where i represents the electrode, can be written as:

$$
G_{i}=c_{i} x_{i}+G_{s}
$$

Equation 2: Slag-to-Matte Conductance 
where $\mathrm{c}$ is the conductance coefficient (in Siemens/m), $\mathrm{x}_{\mathrm{i}}$ is the immersion depth of the electrode in the slag (in $m$ ) and $G_{s}$ is the total conductance of the slag (in S). In other words, $G_{s}$ is the conductance of the slag when the electrodes are positioned at the surface of the slag. Using these assumptions and $\mathrm{KCL}$, it is now possible to solve the EAF electrical circuit.

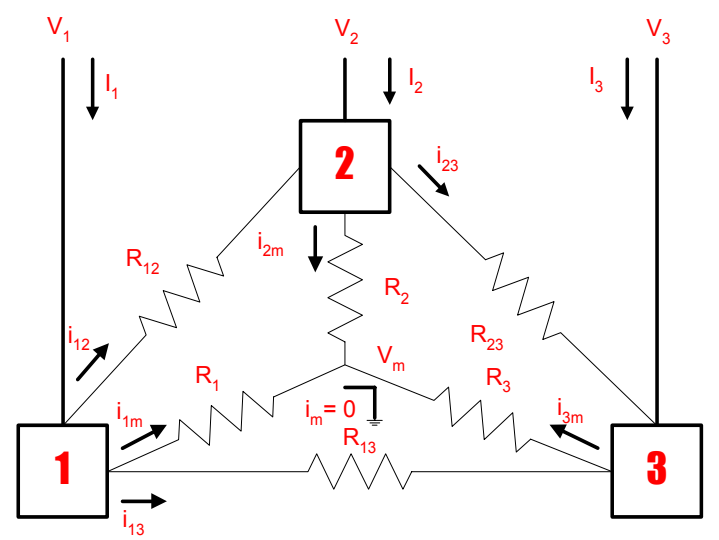

The following sets of equations can be obtained by applying KCL to each of the four nodes displayed in Figure 2.

$$
\begin{gathered}
I_{1}=G_{12}\left(V_{1}-V_{2}\right)+G_{1}\left(V_{1}-V_{m}\right)+G_{13}\left(V_{1}-V_{3}\right) \\
I_{2}=-G_{12}\left(V_{1}-V_{2}\right)+G_{2}\left(V_{2}-V_{m}\right)+G_{23}\left(V_{2}-V_{3}\right) \\
I_{3}=G_{3}\left(V_{3}-V_{m}\right)-G_{13}\left(V_{1}-V_{3}\right)+G_{23}\left(V_{2}-V_{3}\right) \\
G_{1}\left(V_{1}-V_{m}\right)+G_{2}\left(V_{2}-V_{m}\right)+G_{3}\left(V_{3}-V_{m}\right)=0
\end{gathered}
$$

\section{Equation 3: KCL using Conductances}

Equation 4 represents the expression for $\mathrm{V}_{\mathrm{m}}$, which will be used to replace $\mathrm{V}_{\mathrm{m}}$ in the KCL equations above.
Figure 2: Electrical Model of EAF

\subsection{Nodal Equations}

$$
V_{m}=\frac{G_{1} V_{1}+G_{2} V_{2}+G_{3} V_{3}}{G_{1}+G_{2}+G_{3}}
$$

Equation 4: Expression for $V_{m}$

\subsection{Current $\left(I_{I}\right)$ Calculations}

Considering that the currents of the three electrodes will behave in a similar manner, it is not necessary to display in full detail the complete derivation for all three currents. The derivation of $\mathrm{I}_{2}$ and $I_{3}$ therefore follows from $I_{1}$. The final expression for the total current $I_{1}$ flowing through electrode 1 is shown in Eqn 5, with the position inputs properly factored. $I_{1}$ is equal to:

$$
I_{1}=\frac{1}{G_{T O T}}\left[\begin{array}{l}
x_{1}\left(\begin{array}{l}
2 V_{1} c_{1}\left(G_{s}+G\right)-V_{2} c_{1}\left(G_{s}+G\right)-V_{3} c_{1}\left(G_{s}+G\right)+c_{1} c_{2} x_{2}\left(V_{1}-V_{2}\right) \\
+c_{1} c_{3} x_{3}\left(V_{1}-V_{3}\right)
\end{array}\right) \\
+x_{2}\left(V_{1} c_{2}\left(G_{s}+2 G\right)-V_{2} c_{2}\left(G_{s}+G\right)-V_{3} c_{2} G\right) \\
+x_{3}\left(V_{1} c_{3}\left(G_{s}+2 G\right)-V_{2} c_{3} G-V_{3} c_{3}\left(G_{s}+G\right)\right) \\
+\left(2 V_{1}-V_{2}-V_{3}\right)\left(G_{s}^{2}+3 G G_{s}\right)
\end{array}\right]
$$

\section{Equation 5: Current in Electrode $1\left(I_{1}\right)$}

Similarly, Equations 6 and 7 represent the total currents $\mathrm{I}_{2}$ and $\mathrm{I}_{3}$ respectively.

$$
I_{2}=\frac{1}{G_{T O T}}\left[\begin{array}{l}
x_{1}\left(-V_{1} c_{1}\left(G_{s}+G\right)+V_{2} c_{1}\left(G_{s}+2 G\right)-V_{3} c_{1} G\right) \\
+x_{2}\left(\begin{array}{l}
-V_{1} c_{2}\left(G_{s}+G\right)+2 V_{2} c_{2}\left(G_{s}+G\right)-V_{3} c_{3}\left(G_{s}+G\right)+c_{2} c_{3} x_{3}\left(V_{2}-V_{3}\right) \\
+c_{1} c_{2} x_{1}\left(V_{2}-V_{1}\right)
\end{array}\right) \\
+x_{3}\left(-V_{1} c_{3} G-V_{2} c_{3}\left(G_{s}+2 G\right)-V_{3} c_{3}\left(G_{s}+G\right)\right) \\
+\left(-V_{1}+2 V_{2}-V_{3}\right)\left(G_{s}^{2}+3 G G_{s}\right)
\end{array}\right]
$$

\section{Equation 6: Current in Electrode $2\left(\mathrm{I}_{2}\right)$}

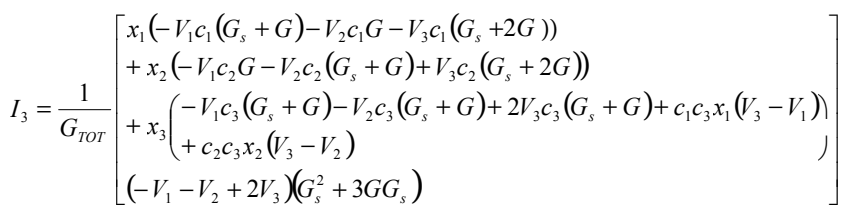

\section{Equation 7: Current in Electrode $3\left(\mathbf{I}_{3}\right)$}

where $G_{T O T}=c_{1} x_{1}+c_{2} x_{2}+c_{3} x_{3}+3 G_{s}$

Equation 8 represents the Current Matrix Model shown by Eqn 1

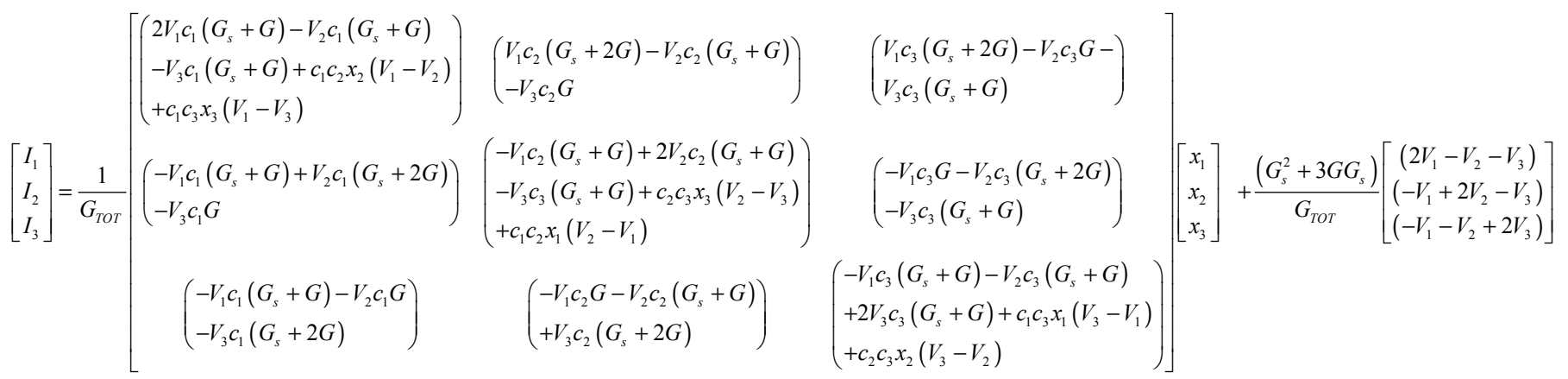

Equation 8: Electrode Current Equations in Matrix Form 
Note that, in Equation 8 above, the $3 \times 3$-conductance matrix is nonlinear. Presence of terms containing $\mathrm{x}_{1}, \mathrm{x}_{2}$, and $\mathrm{x}_{3}$ along the main diagonal indicate this clearly. Therefore this system cannot be controlled as a linear state-space model. Therefore, linearization of the $\mathrm{G}_{\mathrm{ij}}$ conductance matrix will be necessary to control this system with state feedback.

Another observation is that when the electrodes are positioned flush on the slag (i.e. $\mathrm{x}_{1}=\mathrm{x}_{2}=\mathrm{x}_{3}=0$ ), there is still a constant current passing through them. This constant value is represented by the second term of Equation 8. It arises from the presence of $\mathrm{G}_{\mathrm{s}}$ in the slag-to-matte conductance.

\section{$4 \quad$ Initial Model of EAF}

Now that the EAF electrical circuit is solved, the next step is to implement Equation 8 as an open-loop system to test different cases for several electrode positions. For the output current matrix $I_{i}$, this conductance matrix $G_{i j}$ depends on the electrode voltages $\left(\mathrm{V}_{1}, \mathrm{~V}_{2}\right.$ and $\left.\mathrm{V}_{3}\right)$ and their respective conductance coefficients $\left(c_{1}, c_{2}\right.$ and $\left.c_{3}\right)$. Therefore, appropriate values have to be calculated in order implement a realistic system. The chosen values will be explained in the next sub-section.

In addition to setting-up the electrode voltages and conductance coefficients, it is important to set the slag-to-matte conductance offset $\mathrm{G}_{\mathrm{s}}$ and the inter-electrode conductance $\mathrm{G}$. For each of these variables there exists an acceptable range of values capable of adequately modeling the system. Table 1 shows the list of variables with their acceptable ranges and finally the values chosen for simulation purposes.

Table 1: Constants

\begin{tabular}{|c|c|c|}
\hline Variables & $\begin{array}{c}\text { Acceptable } \\
\text { Range }\end{array}$ & Chosen Value \\
\hline \hline $\mathrm{V}_{1}, \mathrm{~V}_{2}$ and $\mathrm{V}_{3}$ & $100-1000 \mathrm{~V}$ & $500 \mathrm{~V}$ \\
\hline $\mathrm{c}_{1}, \mathrm{c}_{2}$ and $\mathrm{c}_{3}$ & $1-100 \mathrm{~S} / \mathrm{m}$ & $20 \mathrm{~S} / \mathrm{m}$ \\
\hline $\mathrm{G}_{\mathrm{s}}$ & $5-25 \mathrm{~S}$ & $10 \mathrm{~S}$ \\
\hline $\mathrm{G}$ & $\approx 0 \mathrm{~S}$ & $0.1 \mathrm{~S}$ \\
\hline
\end{tabular}

Voltages used in the simulation are phasors with $500 \mathrm{~V}$ magnitude and $120^{\circ}$ phase difference. Secondly, all values follow SI units with meters as the length unit.

\subsection{Matlab Simulation}

The Matlab ${ }^{\mathrm{TM}}$ simulations indicate the important values for critical electrode positions. From these simulations, if the electrodes are positioned on the slag, approximately $5 \mathrm{kA}$ of constant current will pass through each of them, while individually using about $2.5 \mathrm{MW}$ of power. When the electrodes are completely immersed in the slag, a maximum of $15 \mathrm{kA}$ of current is present while using a maximum of $7.5 \mathrm{MW}$ per electrode. These simulations also indicate that drastic changes in the position of the electrodes above the slag is less sensitive for current and power as when they are immersed deep in the slag.

\section{Open-Loop Simulations}

\subsection{Simulink Block Diagram}

Figure 3 represents the open-loop block diagram used in the simulation of the EAF.

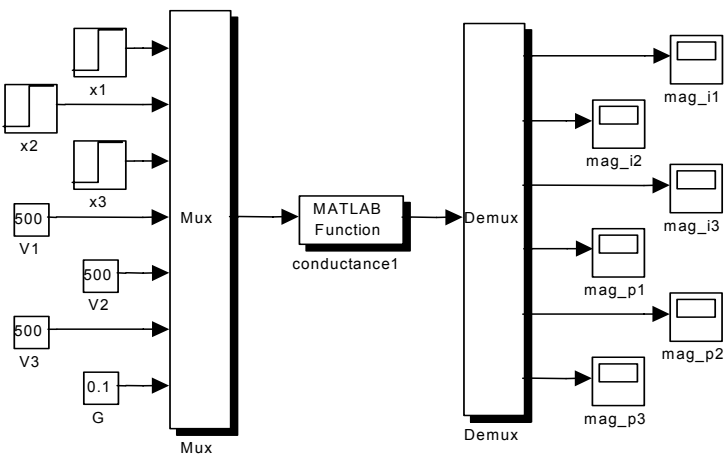

Figure 3: Simulink Open-Loop Block Diagram

As can be seen from Figure 3, there are 7 inputs and 6 outputs to the Matlab function. The position of each electrode $\left(\mathrm{x}_{1}, \mathrm{x}_{2}\right.$, and $\mathrm{x}_{3}$ ) is represented by a step input. The voltages applied to each electrode are represented by constants $V_{1}, V_{2}$ and $V_{3}$ (their phase representation is described within the Matlab code). The last input is the inter-electrode conductance, set to $0.1 \mathrm{~S}$. The outputs are the respective magnitudes of the currents $\left(\mathrm{I}_{1}, \mathrm{I}_{2}\right.$, and $\left.\mathrm{I}_{3}\right)$ and power $\left(\mathrm{P}_{1}, \mathrm{P}_{2}\right.$, and $\left.\mathrm{P}_{3}\right)$ passing through each electrode.

\subsection{Introducing Noise}

In any system, noise plays a prevalent part in altering the output of the system. No system can be modeled efficiently without taking noise into account. The EAF system is no exception. The only difference here is that the noise will not be applied on the input of the system, but rather on the output. The immense size and weight of the electrodes make them immune to small disturbances, and therefore any input noise will have minimal effect on the output. Therefore, any noise affecting the system will have to be introduced on the output side of the system.

\subsection{System Dynamics}

The next step in implementing a controller for the EAF system is to include the system dynamics. Unfortunately, the position step inputs do not realistically represent the inputs to a real-life furnace. In real-life EAFs, hydraulic actuators control the positions of the electrodes [2]. To simplify the physics of the system, an assumption is made that the hydraulic cylinders are powerful enough to neglect the mass of the electrodes. Therefore, the model of the hydraulic system includes a onesecond time delay, followed by a gain (speed conversion), and then an integrator. Figure 4 shows the input half of the openloop system with the proper modifications. 


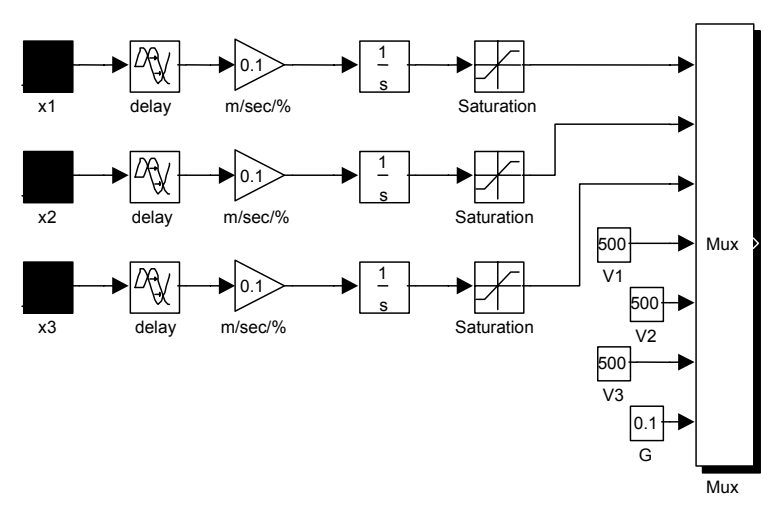

Figure 4: Input Half of Open-Loop incl. System Dynamics

In Figure 4, the input step responses now represent the valve opening as a percentage. The one-second delay represents the actual time it takes for the valves to open/close as desired. The difference between Figures 3 and 4 is that the position of the electrodes is now a function of the percentage valve openings.

Other nonlinear additions to the EAF system include sensors and transducers. Transducers are represented in the system by first order filters. The primary function of transducers however, is not to filter the signal, but to translate the signal from a given input signal to a signal that can be processed. These blocks are placed after the noise in the output half of the open-loop system and are represented by first-order transfer functions with a $25 \mathrm{~ms}$ time constant. Figure 5 shows the location of the transducers in the system.

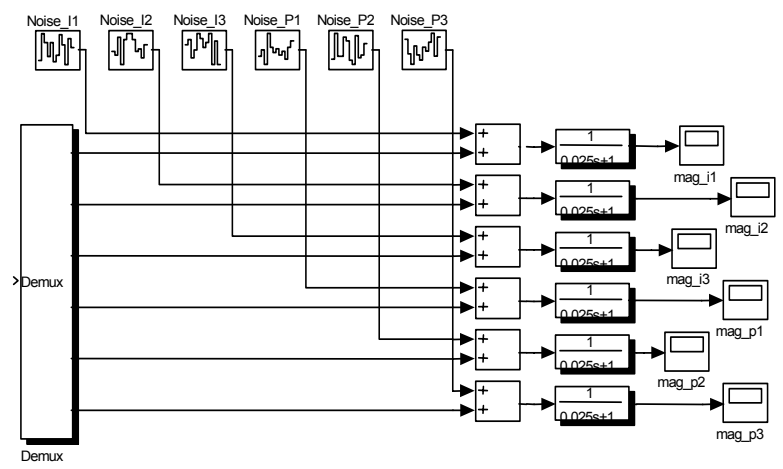

Figure 5: Output Half of Open-Loop with Noise

\section{Control Principle}

One of the main objectives of the three-electrode Electric Arc Furnace simulator study is to have the electrodes maintain constant power consumption. This is achieved by moving the electrodes to a given depth, obtaining the desired resistances (or conductances), which leads to a constant power consumption. To attain this goal, the open-loop system described in the previous section must be closed in order to create an error signal. The control principle is accomplished by minimizing this error signal with specific controllers. For this system, although the power is to remain constant, since the power magnitudes are scalar multiples of the electrode currents, controlling the current will lead to power control as well.

\subsection{Closed-Loop System}

Figure 6 shows the closed-loop system for the EAF.

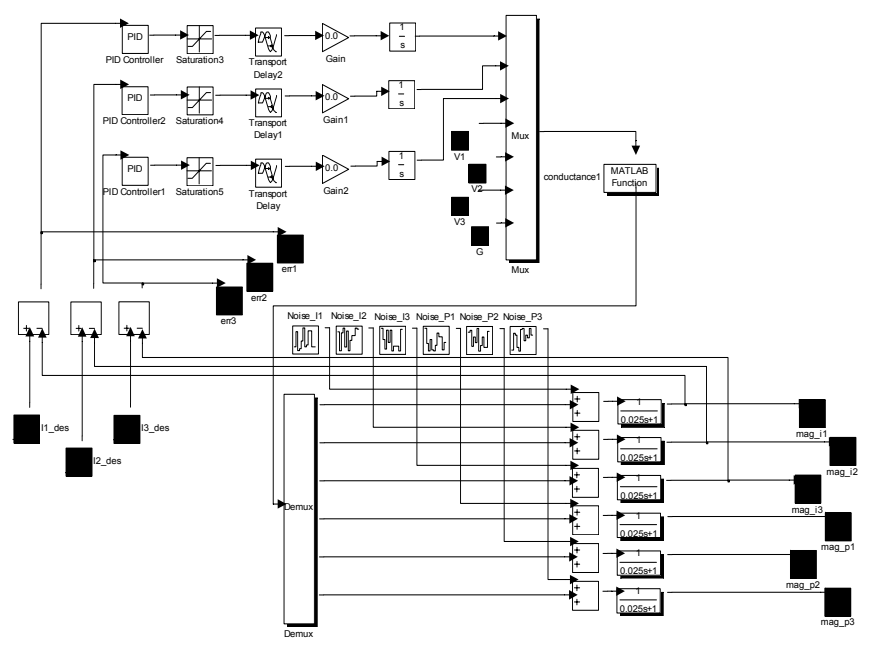

Figure 6: Closed-Loop System

A feedback loop has now been added to the output of the openloop system. The output currents are fed back into the negative port of an adder block, where they are combined with the initial step responses representing the desired current. The difference between the desired current and output current is then fed into a PID controller set appropriately to transform a current magnitude to a percent error. This percent error orders the hydraulic actuators to open the valves such that a new resistance sets the corresponding currents to converge to the desired currents.

The saturation blocks at the top of Figure 6 are relocated preceding the delay and integrator blocks and set to ensure that the percent error never surpasses $\pm 100 \%$. Initially, the step response denoting the desired current is transmitted through the system, activating the electrodes to move up or down. Once the 'Matlab function' block calculates the output current, it is negated and added to the desired current. As the loop is repeatedly executed, the difference between the currents ultimately diminishes to zero and the objective of controlling the power is achieved.

\subsection{Setting the PID Controller}

For preliminary testing, the PID controllers in Figure 6 are fundamentally $\mathrm{P}$ controllers as the D-gain and I-gain are set to 0 . For the purpose of the EAF system, the P-gain had to transform a current signal to a percent error. Thus the gain of the Pcontroller was originally set to 0.001 . However, the closed- loop error took much too long to reach zero (under steady-state conditions).

Therefore, the P controller gain had to be increased to speed up the step response. After much tuning, setting the P-gain to 0.0045 gave the most satisfactory step response. The next subsection will display simulations with different desired currents. 


\subsection{Closed-Loop Simulations}

The first simulation is performed when all the desired currents are set to $10 \mathrm{kA}$, the mean of maximum and minimum electrode currents. Figure 7 represents the output currents. Note that the currents of all electrodes are plotted on the same graph.

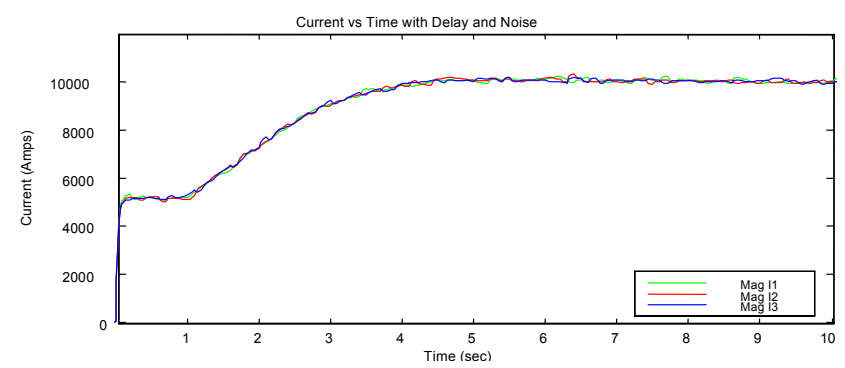

Figure 7: Closed-Loop Current Magnitudes (Desired Currents $=10 \mathrm{kA}$ )

Figure 8 reveals the power magnitudes of the electrodes with the same desired currents as above. As explained previously, the power magnitudes are scalar multiples of the current magnitudes. Hence, the desired power for each electrode is $5 \mathrm{MW}$ for a desired current of 10kA since the magnitude of the voltage is $500 \mathrm{~V}$ RMS.

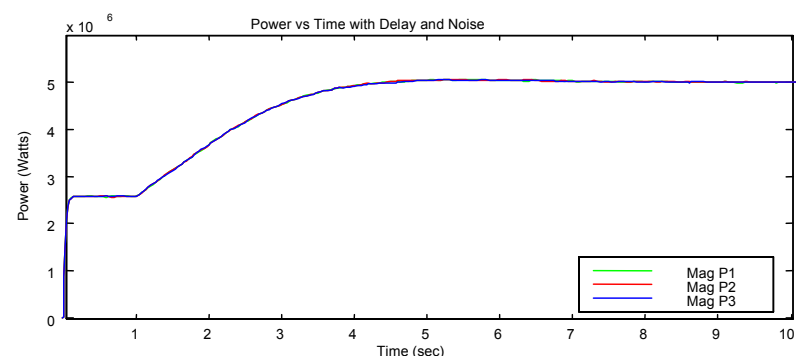

Figure 8: Closed-Loop Power Magnitudes (Desired Power $=$ 5MW)

Since the system model already contains an integrator (in order to change electrode speed into electrode position), the effect of adding an I-gain will be negligible. The next simulation involves testing for coupling of the electrode currents. Figure 9 represents the current magnitude for the case when $\mathrm{I}_{1}=10 \mathrm{kA}$ and $\mathrm{I}_{2}=\mathrm{I}_{3}=$ 5kA. Having different desired currents when running a simulation leads to coupling between the electrodes. This phenomenon is explained by the nonlinear terms found in the conductance matrix $G_{i j}$.

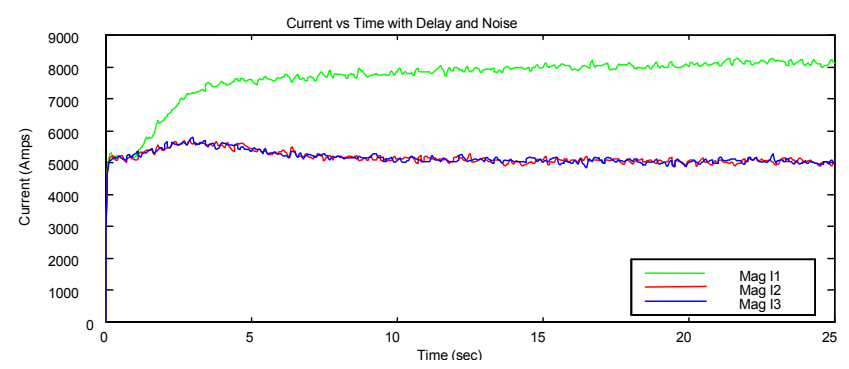

Figure 9: Closed-Loop Current Magnitudes showing Coupling
The consequences of coupling are that the currents have different step responses. By virtue of these differences in error responses, ideal PID controllers would have to monitor the system such that each current attains its steady-state in the fastest time possible. A future goal is to determine a decoupling controller in order to combat these coupling effects.

\section{Conclusion}

The objectives of this study were clearly detailed from the beginning. The EAF electrical circuit was successfully solved and modeled in Matlab. The Matlab simulation determined the system extremes for current flow and power consumption for each electrode. The initial EAF block diagram was designed in Simulink outputting individual current and power magnitudes. Step functions were used for the electrode positions and the Matlab code was transformed into a 'Matlab Function' block in Simulink to perform the necessary calculations.

For a more realistic model of the EAF, the dynamics of the system needed to be included. Hydraulic actuators, sensors and transducers were all appropriately placed in Simulink open-loop system to model electrode movement by valves and the introduction of noise. Several simulations were executed and plotted representing a wide range of electrode displacements.

Finally, a feedback loop was introduced to create a closed-loop system. The input step functions now represent the desired current to control the power. The current output was fed back and subtracted from the desired current to form an error signal. The error signal was transformed to a percent error by a PID controller. All simulations demonstrated throughout the project implemented a P-controller with a 0.0045 gain. Adding the differentiator and integrator gains will improve the step response. The coupling effects between electrodes were also examined.

Future goals for the three-electrode arc furnace simulator study include further testing for all coupling currents, the development of optimal decoupling controllers, and linearizing the system in order to implement a state-space controller. Analysis of many parameters' effects on the EAF model can also be included in future objectives. These parameters can include the effect of the reactances on the system's power factor and the effect of arcing as a function of temperature inside the furnace. Another objective would be to see if the ideal PID-regulators for linearcontrolled electrodes would work in 'bang-bang' furnaces and if not, find the relationship between the two types of furnaces.

\section{References}

[1] B. Boulet, V. Vaculik, G. Wong, Control of High-Power Non-Ferrous Smelting Furnaces, IEEE Canadian Review, summer 1997.

[2] G. Dosa, A. Kepes, T. Ma and P. Fantin, Computer control of high-power electric furnaces. Challenges in Process Intensification Symposium, 35th Conference of Metallurgists of the Metallurgical Society of CIM, Montreal, Quebec, August 24-29, 1996. 